\section{Measurement of Piezoelectric Properties of Pulsed Laser Deposited Hydroxyapatite Thin Films on Platinum or Titanium Substrate}

Tsutomu Nishigaki, Hiroaki Nishikawa, Masanobu Kusunoki and Shigeki Hontsu

Faculty of Biology-Oriented Science and Technology, Kinki University, 930 Nishimitani, Kinokawa, Wakayama 649-6493, Japan

\begin{abstract}
In order to measure the piezoelectric properties of the Hydroxyapatite (HAp) films, we have fabricated $\mathrm{Cu} / \mathrm{HAp} / \mathrm{T}$ or $\mathrm{Cu} / \mathrm{HAp} / \mathrm{Pt}$ structure. At first, a $1.5 \mathrm{~m}$ thick HAp was deposited on a Ti or Pt substrate using the KrF Pulsed Laser Deposition (PLD) method. After the HAp deposition, the HAp film was crystallized by post-annealing in nitrogen gas atmosphere and cooled slowly in an electric furnace. Then, a Cu top electrode sheet was attached on HAp film. Finally, one end of the $\mathrm{Cu} / \mathrm{HAp} / \mathrm{Ti}$ or $\mathrm{Cu} / \mathrm{HAp} / \mathrm{Pt}$ structure was clamped to compose a vibrating cantilever beam. Piezoelectric coefficients were estimated by output voltage responses of HAp films measured by a operational amplifier circuit when the $\mathrm{Cu} / \mathrm{HAp} / \mathrm{Ti}$ or $\mathrm{Cu} / \mathrm{HAp} / \mathrm{Pt}$ beam was excited by a mini-shaker at the first natural frequency of the beam. The results showed the piezoelectricity of the artificially synthesized HAp films.
\end{abstract}

Keywords: Hydroxyapatite; Piezoelectricity; Pulse laser deposition; Post-annealing; Vibration response; Cantilever beam

\section{Introduction}

It has been well known that bone has piezoelectric properties and it has been said that this properties affect the process of growth and the remodeling of bone. These piezoelectric properties of the bone was considered to be caused by the shift of the center of symmetry of the positive and negative electrical charge due to the strain of the collagen fibers included in the bone. Thus, it has been considered that there were no piezoelectric effects in the hexagonal HAp which has center of symmetry of crystal. However, in recent years, the piezoelectric property of artificially synthesized HAp was reported [1]. In the artificially synthesized HAp, there were no longer any perfect center of symmetry of crystal, and the piezoelectricity is considered to be caused by the shift of the center of symmetry of the positive and negative electrical charge due to the strain of the HAp itsef. In this study, in order to measure the piezoelectric properties, HAp thin films wSere deposited on a Ti or Pt substrate using PLD method, anSd piezoelectric properties of the HAp film were estimated by measuring vibration responses of the mechanical strains and electrical voltages of the HAp structure simultaneously.

\section{Materials and Methods}

\section{Deposition of HAp film}

The HAp films were fabricated by PLD method using an $\mathrm{KrF}$ excimer laser (Lambda Physik LPX-210icc; $\lambda=248 \mathrm{~nm}$; full width at half maximum of pulse $=20 \mathrm{~ns}$ ) [2]. Schematic diagram of PLD method is shown in Figure 1. The HAp bulk (CELLYARDTM pellet) was placed in the vacuum chamber (background pressure of approximately $8 \times$ $10^{-7}$ Torr). Molecules, atoms, and ions consisting of the target surface were desorbed in the vacuum chamber by irradiation with and $\mathrm{KrF}$ excimer laser beam and react with the atmosphere gas $\left(\mathrm{O}_{2}+\mathrm{H}_{2} \mathrm{O}\right.$, partial pressure was approximately $0.8 \mathrm{mTorr}$ ) on the Ti or Pt substrate, where the size of substrate was $80 \mathrm{~mm}$ long, $10 \mathrm{~mm}$ width and $0.3 \mathrm{~mm}$ thick. The laser energy density on the target was approximately $1 \mathrm{~J} / \mathrm{cm}^{2}$ and the pulse repetition rate was $10 \mathrm{~Hz}$. The film deposition rate was about $10 \mathrm{~nm} / \mathrm{min}$. The total thickness of the HAp films was $1.5 \mu \mathrm{m}$.

X-ray Diffraction (XRD) showed that as-deposited HAp films were amorphous. Therefore, after deposition, a heat treatment was necessary to convert the disordered structure of the film to a properly crystallized form. This post-annealing was carried out at $350^{\circ} \mathrm{C}$ for 5 hours in

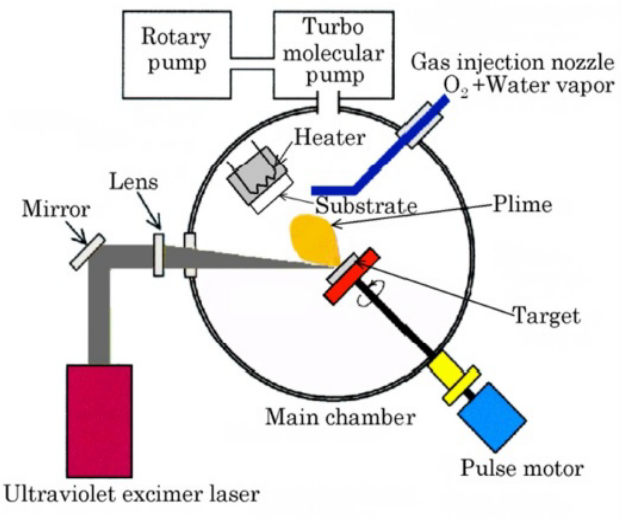

Figure 1: Schematic diagram of PLD method.

nitrogen gas atmosphere and cooled slowly in an electric furnace. XRD patterns of the HAp film deposited on titanium or platinum substrate after annealing at $350^{\circ} \mathrm{C}$ are shown in Figure 2. From these figures, it is observed that post-annealing process was more effective against HAp film deposited on Pt substrate than HAp film on Ti substrate. The reason of this difference may due to the oxidized layer produced between the surface of the Ti substrate and HAp films.

A number of HAp/Ti substrates was fabricated and post-annealed to measure the break down voltages of HAp films. The voltage was applied between Pt substrate and the electrode of evaporated gold on the surface of HAp. Measured break down voltages and estimated break down electric field of typical three specimens were shown in

*Corresponding author: Tsutomu Nishigaki, Faculty of Biology-Oriented Science and Technology, Kinki University, 930 Nishimitani, Kinokawa, Wakayama 6496493, Japan, E-mail: nisigaki@waka.kindai.ac.jp

Received June 08, 2013; Accepted July 23, 2013; Published August 23, 2013

Citation: Nishigaki T, Nishikawa H, Kusunoki M, Hontsu S (2013) Measurement of Piezoelectric Properties of Pulsed Laser Deposited Hydroxyapatite Thin Films on Platinum or Titanium Substrate. Bioceram Dev Appl S1: 008. doi: 10.4172/20905025.S1-008

Copyright: (C) 2013 Nishigaki T, et al. This is an open-access article distributed under the terms of the Creative Commons Attribution License, which permits unrestricted use, distribution, and reproduction in any medium, provided the original author and source are credited. 
Table 1. It was shown that estimated break down electric field was about $2.8 \times 10^{5}$ to $3.0 \times 10^{5} \mathrm{~V} / \mathrm{cm}$. However, short circuit between Pt or Ti substrate and the electrode of evaporated gold on the surface of HAp occurred in some specimens. As shown in Figure 3, by using Scanning Electron Microscope (SEM), microcrack formations were observed in HAp after post-annealing due to the difference of the coefficient of thermal expansion between HAp and Ti or Pt. Here, coefficients of thermal expansion of Ti, Pt and HAp are 8.6, 8.8 and $13.7\left(\times 10^{-6} \mathrm{~K}^{-1}\right)$. To avoid the short circuit, finally, a $70 \mu \mathrm{m}$ thick $\mathrm{Cu}$ top electrode sheet was attached on the HAp film instead of evaporated gold. This layered configuration of $\mathrm{Cu} / \mathrm{HAp} / \mathrm{Ti}$ or $\mathrm{Cu} / \mathrm{HAp} / \mathrm{Pt}$ enabled the verification of piezoelectric property of HAp films.

\section{Estimation method of the piezoelectricity of HAp}

Based on the structural vibration engineering technique, piezoelectricity of the thin sheet-like materials can be estimated from
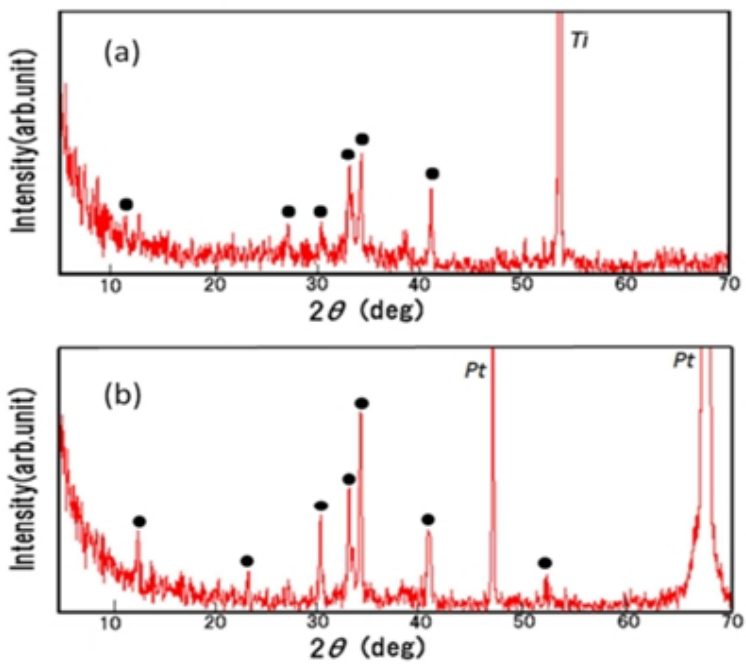

Figure 2: XRD patterns of (a) the HAp film deposited on titanium or (b) the HAp film deposited on platinum substrate (the peaks corresponding to the HAp films are denoted by black dots).

\begin{tabular}{|c|c|c|c|}
\hline & Break down voltage & \multicolumn{2}{|c|}{ Electric field } \\
\hline & $(\mathrm{V})$ & \multicolumn{2}{|c|}{$(\mathrm{V} / \mathrm{cm})$} \\
\hline Specimen \#1 & 45 & 3.0 & 10 \\
\hline Specimen \#2 & 42 & 2.8 & 10 \\
\hline Specimen \#3 & 42 & 2.8 & 10 \\
\hline
\end{tabular}

Table 1: Measured break down voltages and electric field of HAp films of thickness $1.5 \mathrm{~m}$ deposited on $10 \mathrm{~mm}$ square Ti substrate.

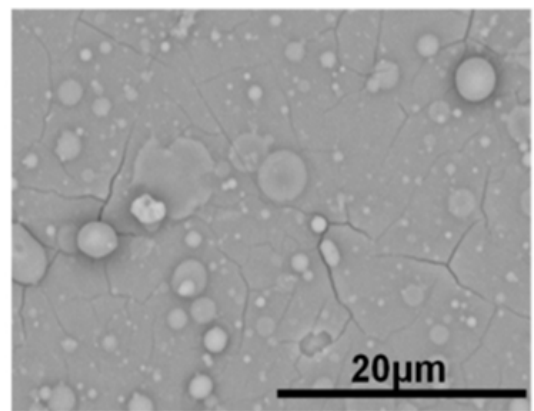

Figure 3: Microcrack formation of HAp observed by S EM. the vibration response of the structure which contains piezoelectric elements. In this study, vibration of flexible cantilever beam is used to measure the piezoelectric property of the HAp films. The analytical model and the coordinate system are shown in Figure 4. A piezoelectric film of length $L$, width $b$ and thickness $t$ is perfectly bonded on a cantilever beam of length $l$, width $b$ and thickness $t_{B}$. A Cartesian coordinate system o- $x y z$ is defined and the origin is located at the center of one end section of the beam. Based on the Hamilton's principle, equations of motion of the beams were derived as follows:

$$
\rho \mathrm{A} \frac{\partial^{2} y}{\partial x^{2}}+\frac{\partial^{2}}{\partial x^{2}}\left(\mathrm{E}_{B} I \frac{\partial^{2} u}{\partial x^{2}}-\sum_{i=1}^{N} M_{(i)}\right)=0
$$

where, $\rho, A, u, E_{B}$ and $I$ are density, cross section, bending displacement, Young's modulus and moment of inertia of the beam, and $M_{(i)}$ are the bending moments applied to the beam caused by the converse piezoelectric effect [3]. $N$ denote the number of piezoelectric films attached on the beam (i.e. $N=1$ in this study). The output voltages $V_{S}$ of the piezoelectric materials are expressed as follows:

$$
V_{S}=\frac{1}{2} \frac{t_{C} t_{B}}{L} \frac{d_{31} E_{P}}{\in}\left(\left.\frac{\partial u}{\partial x}\right|_{x_{2}}-\left.\frac{\partial u}{\partial x}\right|_{x_{1}}\right)
$$

where, $E_{P}$ and $\varepsilon$ are Young's modulus and dielectric constants of the piezoelectric films, and $x_{1}$ and $x_{2}$ are the positions of both endpoints of the HAp film along x-axis. From equation (2), Piezoelectric constant $d_{31}$ is expressed as follows:

$$
d_{31}=\frac{2 L \in V_{S}}{t_{C} t_{B} E_{P}} \frac{1}{\left\{\phi_{j}{ }^{\prime}\left(x_{2}\right)-\phi_{j}{ }^{\prime}\left(x_{1}\right)\right\}} \frac{\phi_{j}}{u(l)}
$$

where, $\phi_{j}(x)$ are normalized mode shape functions of the BernoulliEuler beam which satisfy geometrical boundary conditions and prime represent derivatives with respect to the coordinate $\mathrm{x}$. In equation (3), $L, t_{C}, t_{B}$ and $\phi_{j}(x)$ are known constants and functions as they are measurable or analytically derived. Therefore, once if Young's modulus and dielectric constants of the HAp were given, from equation (3), the unknown piezoelectric constant $d_{31}$ can be estimated from the measured output voltages $V_{S}$ of the piezoelectric films and tip

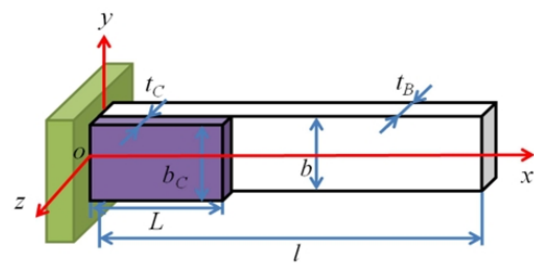

Figure 4: Analytical model and coordinate system.

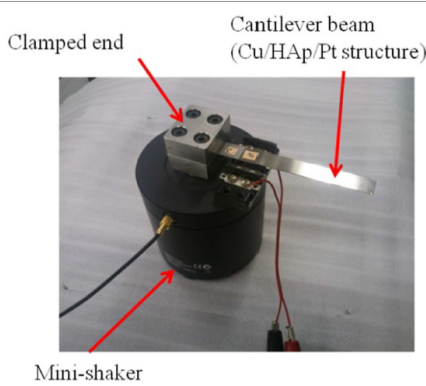

Figure 5: Experimental set-up for measuring vibration response of $\mathrm{Cu}$ / $\mathrm{HAp} / \mathrm{Pt}$ cantilever beam. 
displacement $u(l)$ of the beams. In the experiment, one end of the beam was clamped within $20 \mathrm{~mm}$ range from the tip by two steel plate of thickness $10 \mathrm{~mm}$ to compose a vibrating cantilever beam. As shown in Figure 5, the clamped end of the beam was mounted on the moving stage of the small vibration exciter (mini-shaker, B\&K 4810). The minishaker was driven at the first resonance frequency of the cantilever beam. In the resonance state, the amplitude of the clamped end of the beam was small enough compared with the amplitude of the free end, thus only the tip displacement responses of the beam were measured by a laser displacement sensor. In the measurement of piezoelectric output voltages, impedance matching between output resistance of the piezoelectric materials and input resistance of the measuring equipment is significant for the accuracy of the measured voltages. Therefore, a voltage follower circuit composed by a operational amplifier was used and was directly connected to the electrodes of the HAp film.

\section{Results and Discussion}

Time histories of measured output voltages of HAp at the initial state (i.e. the beam was at rest) and in the case when the beam was excited at the first resonance frequency of the beam $(16.6 \mathrm{~Hz})$ are shown in Figure 6. In each figure, horizontal axis stands for time (sec) and vertical axis stands for output voltages (V). In the measurement of output voltages, unwanted noise in the very low frequency area (under $1 \mathrm{~Hz}$ ) and also hum noise from AC power supply $(60 \mathrm{~Hz})$ were observed. As the frequencies of these unwanted signals are far enough from the excitation frequency, a band-pass filter was used to eliminate these noise signals from the measured voltage responses. By comparing these two figures, it is clear that, in the output signal of the HAp film, sine wave signal correspond to the mechanical strain of the surface of the beam was clearly observed. It should be pointed out here that it was confirmed in the experiment that these sine wave signal vanished when the resonance vibration of the beam was physically restrained. Thus, it is obvious that the artificially synthesized HAp films certainly
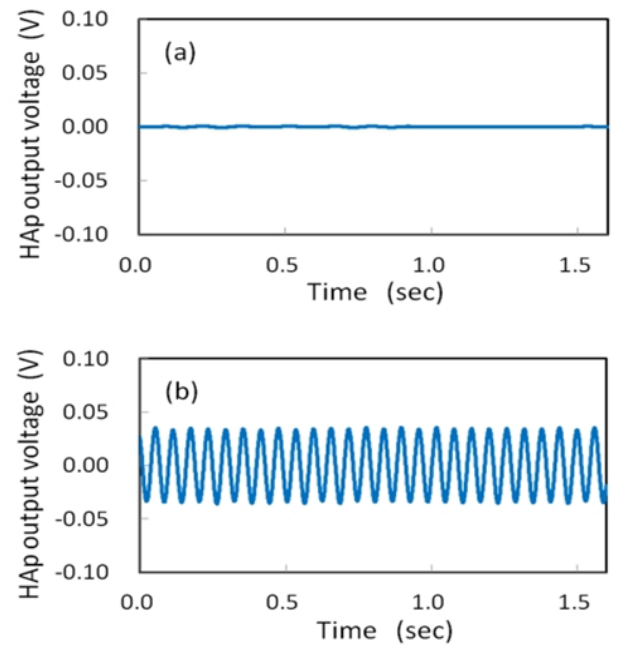

Figure 6: Time histories of measured output voltages of HAp at (a) the initial state (i.e. the beam was at rest) and in (b) the case when the beam was excited at the first resonance frequency $(16.6 \mathrm{~Hz})$. show piezoelectric effect. Though the details are not shown in this paper, roughly estimated piezoelectric constant $\mathrm{d} 31$ from the result of this experiment was in the order of $0.1 \mathrm{pC} / \mathrm{N}$. Further experiments are needed to verify the reproducibility of these measurement results, the piezoelectric converse effect, and the piezoelectric properties after poling process of the artificially synthesized HAp films.

\section{Conclusion}

Thin HAp flims were deposited by using PLD method to compose $\mathrm{Cu} / \mathrm{HAp} / \mathrm{Ti}$ or $\mathrm{Cu} / \mathrm{HAp} / \mathrm{Pt}$ structure and were crystallized by postannealing process. From the XRD patterns, it was observed that the HAp film of superior property was deposited not on Ti substrate but on Pt substrate due to the lack of oxidized layer between HAp and $\mathrm{Pt}$ substrate. Break down voltages of HAp films were also measured and estimated break down electric field was about $2.8 \times 10^{5}$ to $3.0 \times 10^{5} \mathrm{~V} / \mathrm{cm}$. It was shown by using SEM that microcrack formation was observed in HAp after post-annealing due to the difference of the coefficient of thermal expansion between HAp and Pt. Due to the microcrak, short circuit between Pt substrate and the electrode of evaporated gold on the surface of HAp occurred. To avoid the short circuit, finally, a $\mathrm{Cu}$ top electrode sheet was attached on the HAp film instead of evaporated gold. Finally, piezoelectric coefficients were estimated by output voltage responses of HAp films measured by a operational amplifier circuit when the $\mathrm{Cu} / \mathrm{HAp} / \mathrm{Pt}$ beam was excited by a mini-shaker at the first natural frequency of the beam. It was verified that the HAp film possesses piezoelectricity, and further experiments were needed to establish the fabrication method of HAp films with excellent piezoelectric properties, which are currently ongoing projects.

\section{References}

1. Lang SB, Tofail SAM, Gandhi AA, Gregor M, Wolf-Brandstetter C, et al. (2011) Pyroelectric, piezoelectric, and photoeffects in hydroxyapatite thin films on silicon. Appl Phys Lett 98: 123703.

2. Nishikawa H, Hatanaka R, Kusunoki M, Hayami T, Hontsu S (2008) Preperation of freestanding hydroxyapatite membranes with excellent biocompatibility and flexibility. Appl Phys Exp 1: 088001.

3. Nishigaki T (2008) A new shaping design method of distributed piezoelectric film sensor/actuator for vibration control of flexible beams. Mem School B.O.S.T Kinki Univ 22: 1-17. 